\begin{tabular}{|c|l|}
\hline Title & Conductance spectroscopy of spin-triplet superconductors. \\
\hline Author(s) & A sano, Y asuhiro; Tanaka, Y ukio; Golubov, A lexander A.; Kashiwaya, Satoshi \\
\hline Citation & $\begin{array}{l}\text { Physical Review Letters, 99(6), 067005 } \\
\text { https://doi.org/10.1103/PhysRevLett.99.067005 }\end{array}$ \\
\hline Issue Date & 2007-08-10 \\
\hline Doc URL & http://hdl.handle.net/2115/29633 \\
\hline Rights & Copyright $\odot 2007$ A merican Physical Society \\
\hline Type & article \\
\hline File Information & PRL99-6.pdf \\
\hline
\end{tabular}

Instructions for use 


\title{
Conductance Spectroscopy of Spin-Triplet Superconductors
}

\author{
Yasuhiro Asano, ${ }^{1}$ Yukio Tanaka, ${ }^{2}$ Alexander A. Golubov, ${ }^{3}$ and Satoshi Kashiwaya ${ }^{4}$ \\ ${ }^{1}$ Department of Applied Physics, Hokkaido University, Sapporo 060-8628, Japan \\ ${ }^{2}$ CREST-JST and Department of Applied Physics, Nagoya University, Nagoya 464-8603, Japan \\ ${ }^{3}$ Faculty of Science and Technology, University of Twente, 7500 AE, Enschede, The Netherlands \\ ${ }^{4}$ National Institute of Advanced Industrial Science and Technology, Tsukuba 305-8568, Japan
}

(Received 2 May 2007; published 10 August 2007)

\begin{abstract}
We propose a novel experiment to identify the symmetry of superconductivity on the basis of theoretical results for differential conductance of a normal metal connected to a superconductor. The proximity effect from the superconductor modifies the conductance of the remote current depending remarkably on the pairing symmetry: spin singlet or spin triplet. The clear-cut difference in the conductance is explained by symmetry of Cooper pairs in a normal metal with respect to frequency. In the spin-triplet case, the anomalous transport is realized due to an odd-frequency symmetry of Cooper pairs.
\end{abstract}

DOI: 10.1103/PhysRevLett.99.067005

PACS numbers: 74.50.+r, 74.25.Fy, 74.70.Tx

The clear distinction between spin-singlet and spintriplet superconductors is currently a challenging issue in condensed matter physics. Several experimental methods can be used for this purpose: nuclear magnetic resonance, muon spin rotation, critical magnetic field beyond the Pauli limit, Josephson $\pi$ junctions, thermal conductivity, and the detection of multiple phases by spin susceptibility and specific heat. For instance, the unchanged Knight shift across the critical temperature $T_{c}$ suggests spin-triplet superconductivity. This result, however, is not a sufficient condition for spin-triplet superconductivity because spinsinglet superconductivity with strong spin-orbit coupling may also explain the unchanged Knight shift. In addition, the complicated procedure of data analysis may make the conclusion unclear. Other experiments also involve such unclear factors. At present, only a series of different experiments can lead to the conclusion about spin-triplet superconductivity as in the case of $\mathrm{Sr}_{2} \mathrm{RuO}_{4}$ [1]. The experimental methods listed above involve an applied magnetic field. Such experiments are powerless to analyze ferromagnetic superconductors such as $\mathrm{UGe}_{2}$ and $\mathrm{URhGe}$ [2] because the magnetic moment of a superconductor spoils an experimental signal. However, these compounds are undoubtedly promising candidates for spin-triplet superconductivity. To find a way out of the stalemate, one has to use intrinsic phenomena related to spin-triplet superconductivity. We address this issue in the present Letter.

In normal-metal-superconductor (NS) junctions, Cooper pairs penetrate from a superconductor into a normal metal. This phenomenon is called the proximity effect and is very sensitive to the pairing symmetry of a superconductor [3-6]. In junctions with spin-triplet superconductors, Cooper pairs penetrating into a normal metal have odd-frequency symmetry [7]. Although odd-frequency superconductivity itself has never been confirmed in any material, the proximity effect involving odd-frequency pairs is currently a hot topic [7-10]. The existence of odd-frequency pairs causes the drastic enhancement of the quasiparticle density of states (DOS) at Fermi energy in a normal metal $[10,11]$. We will show in this Letter that the enhancement of the DOS can be detected as a zero-bias anomaly in the differential conductance of a proximity structure as shown in Fig. 1. In the last decade, several authors discussed an interesting feature of the proximity effect on the remote electric current in similar junctions of the $s$-wave superconductor $[12,13]$. We will also show that conductance spectra always have a dip structure around zero-bias for all spin-singlet superconductor junctions. On the basis of the robustness of the phenomena, we will conclude that the conductance spectroscopy may serve as a useful tool to test spin-triplet superconductivity. This

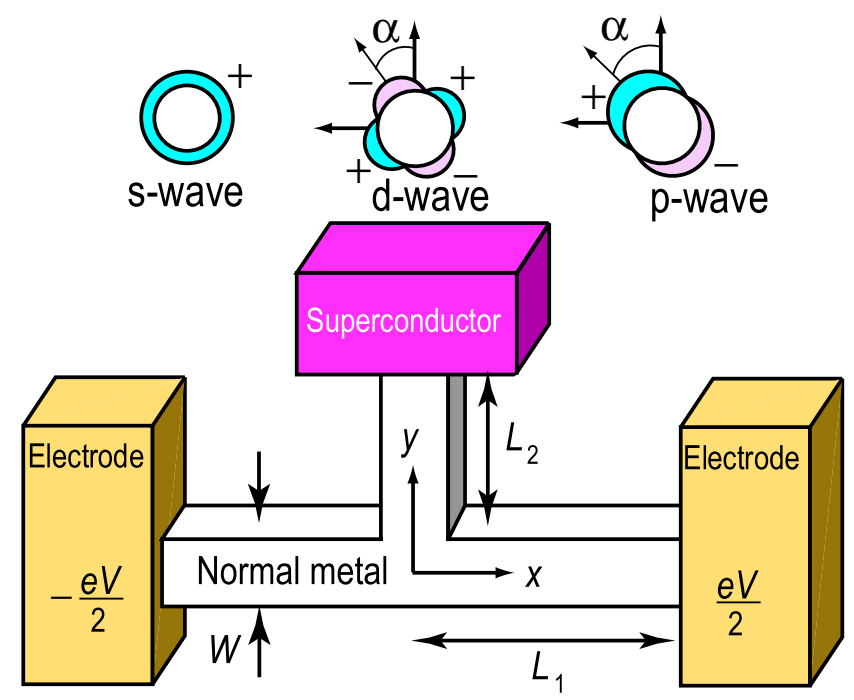

FIG. 1 (color online). Schematic figures of a $T$-shaped proximity structure and pair potentials on the Fermi surface. 
discussion connects the physics of mesoscopic transport and that of unconventional superconductivity.

Let us consider the $T$-shaped junction as shown in Fig. 1. Bias voltage $e V$ is applied to the horizontal normal metal which is connected with two electrodes at $x= \pm L_{1}$. The normal metal has the third branch which is terminated by a superconductor at $y=L_{2}$. To calculate the conductance of a normal metal, we solve the quasiclassical Usadel equation [14] in the Keldysh formalism,

$$
\begin{gathered}
\hbar D \nabla\{\check{G}(\boldsymbol{r}) \nabla \check{G}(\boldsymbol{r})\}+i[\check{H}, \check{G}(\boldsymbol{r})]_{-}=0, \\
\check{G}(\boldsymbol{r})=\left(\begin{array}{cc}
\hat{g}^{R}(\boldsymbol{r}) & \hat{g}^{K}(\boldsymbol{r}) \\
\hat{0} & \hat{g}^{A}(\boldsymbol{r})
\end{array}\right), \quad \check{H}=\left(\begin{array}{cc}
\epsilon \hat{\tau}_{3} & \hat{0} \\
\hat{0} & \boldsymbol{\epsilon} \hat{\tau}_{3}
\end{array}\right),
\end{gathered}
$$

where $D$ is the diffusion constant of a normal metal, $\epsilon$ is the energy of a quasiparticle measured from the Fermi level, and $\hat{\tau}_{i}$ for $i=1-3$ are the Pauli matrices. The symbols $\cdot \hat{i}$. and $\div$ indicate $2 \times 2$ and $4 \times 4$ matrices, respectively. In the following, we solve the Usadel equation in two dimensions. The results are valid also for three-dimensional junctions shown in Fig. 1. We assume that a spin-triplet Cooper pair consists of two electrons with opposite spin directions. This assumption does not break the generality of the following discussion. The Keldysh Green function can be decomposed by $\hat{g}^{K}=\hat{g}^{R} \hat{h}-\hat{h} \hat{g}^{A}$ with $\hat{h}=f_{L}+$ $f_{T} \hat{\tau}_{3}$, where $f_{L}$ and $f_{T}$ are the distribution function of a quasiparticle. From the Keldysh part of Eq. (1), we derive the modified diffusion equation which describes the kinetics of a quasiparticle in a normal metal $[15,16]$,

$$
\nabla\left(D_{T} \nabla f_{T}\right)=0,
$$

with $D_{T}=\operatorname{Tr}\left(1-\hat{g}^{R} \hat{\tau}_{3} \hat{g}^{A} \hat{\tau}_{3}\right) / 4$. The electric current defined by $I=e N_{0} D \int_{-\infty}^{\infty} d \epsilon J_{T}$ can be calculated from the integration of Eq. (3) along $\boldsymbol{r}=(0,0)$ to $\boldsymbol{r}=\left(L_{1}, 0\right)$,

$$
J_{T}=\frac{F_{R}}{L_{1}^{-1} \int_{0}^{L_{1}} d x D_{T}^{-1}},
$$

where we apply the boundary conditions as

$$
f_{T}\left(x=L_{1}\right)=F_{R}=\frac{1}{2}\left\{\tanh \left(\frac{\epsilon_{+}}{2 T}\right)-\tanh \left(\frac{\epsilon_{-}}{2 T}\right)\right\}
$$

with $\epsilon_{ \pm}=\epsilon \pm \mathrm{eV} / 2$ and $T$ being a temperature. At $x=0$, we also apply $f_{T}=0$. At the cross point $\boldsymbol{r}=(0,0)$, the current conservation law implies $\left.\sum_{i} \boldsymbol{n}_{i} \check{G} \nabla_{i} \check{G}\right|_{r=0}=0$, where $\boldsymbol{n}_{i}$ is the unit vector points to outside of the cross point [17]. The retarded part of the Usadel equation is expressed by the usual $\theta$ parametrization

$$
\hbar D \nabla^{2} \theta(\boldsymbol{r})+2 i \epsilon \sin \theta(\boldsymbol{r})=0 .
$$

We find the relation $D_{T}=\cosh ^{2}[\operatorname{Im} \theta(\boldsymbol{r})]$. The Usadel equation is supplemented by the boundary condition at $\boldsymbol{r}=$ $\left(0, L_{2}\right)$ which depends on the pairing symmetry of a superconductor $[4,6,18]$,

$$
\begin{gathered}
\left.\frac{\partial \theta(0, y)}{\partial y}\right|_{r=\left(0, L_{2}\right)}=\frac{\rho_{N}}{W} \frac{\langle F\rangle}{R_{B} T_{B}} \\
\langle F\rangle=\int_{-\pi / 2}^{\pi / 2} d \gamma \frac{T_{N} \cos \gamma\left(f_{s} \cos \theta_{0}-g_{s} \sin \theta_{0}\right)}{\left(2-T_{N}\right) \xi+T_{N}\left(g_{s} \cos \theta_{0}+f_{s} \sin \theta_{0}\right)},
\end{gathered}
$$

where $T_{N}=\cos ^{2} \gamma /\left(z_{0}^{2}+\cos ^{2} \gamma\right), \gamma$ is the incident angle of a quasiparticle measured from the $y$ axis, $\theta_{0}=\theta\left(0, L_{2}\right)$, and $\rho_{N}$ is the resistivity of a normal metal. We assume a potential barrier $z_{0} \hbar v_{F} \delta\left(y-L_{2}\right)$ at the NS interface with $v_{F}$ being the Fermi velocity. The transmission probability and the resistance of the NS interface are given by $T_{B}=$ $\int_{0}^{\pi / 2} d \gamma \cos \gamma T_{N}$ and $R_{B}=\left[\left(2 e^{2} / h\right)\left(k_{F} W\right) T_{B} / \pi\right]^{-1}$, respectively. The Green function in a superconductor depends on $\gamma$ and the orientation angle $\alpha$ in Fig. 1 as $g_{ \pm}=\epsilon / \sqrt{\epsilon^{2}-\Delta_{ \pm}^{2}}$ and $f_{ \pm}=\Delta_{ \pm} / \sqrt{\Delta_{ \pm}^{2}-\epsilon^{2}}$, where $\Delta_{ \pm}=\Delta_{0} \Psi\left(\gamma_{ \pm}\right)$with $\Delta_{0}$ being the amplitude of the pair potential at $T=0, \gamma_{+}=\gamma-\alpha$ and $\gamma_{-}=\pi-\gamma-\alpha$. The form factor $\Psi(\gamma)$ characterizes pairing symmetry as $\Psi(\gamma)=1, \cos \gamma$, and $\cos 2 \gamma$ for $s-, p$-, and $d$-wave symmetries, respectively. In Eq. (7), $g_{s}=\left(g_{+}+g_{-}\right), \xi=1+$ $g_{+} g_{-}+f_{+} f_{-}$, and $f_{s}=\left(f_{+}+f_{-}\right)$for the spin-singlet pairing symmetry and $f_{s}=i\left(f_{+} g_{-}-f_{-} g_{+}\right)$for the spintriplet one $[4,6]$. At $x= \pm L_{1}$, we impose $\theta\left( \pm L_{1}, 0\right)=0$. The differential conductance at zero temperature results in

$$
\left.R_{N} \frac{d I}{d V}\right|_{e V}=\left.\left[\frac{1}{L_{1}} \int_{0}^{L_{1}} \frac{d x}{\cosh ^{2}[\operatorname{Im} \theta(x, 0)]}\right]^{-1}\right|_{\epsilon=e V},
$$

where $R_{N}=2 L_{1} \rho_{N} / W$ is the normal state resistance of the junction. In what follows, we fix the Thouless energy of a half horizontal wire $E_{\text {th }} \equiv \hbar D / L_{1}^{2}$ at $0.04 \Delta_{0}$.

First we discuss the differential conductance of the spintriplet $p$-wave junctions in Fig. 2(a), where $R_{N} / 2 R_{B}=1$, $z_{0}=1$, and $\alpha=0$. The conductance has strong zero-bias peak. The width of the zero-bias conductance peak (ZBCP) decreases with increasing $L_{2}$ because the energy $\hbar D /\left(L_{1}+L_{2}\right)^{2}$ characterizes the peak width. Thus one should fabricate $L_{2}$ as short as possible to observe the ZBCP in experiments. In what follows, we fix $L_{2} / L_{1}=$ 0.1 . On the other hand, the height of the ZBCP is indepen-
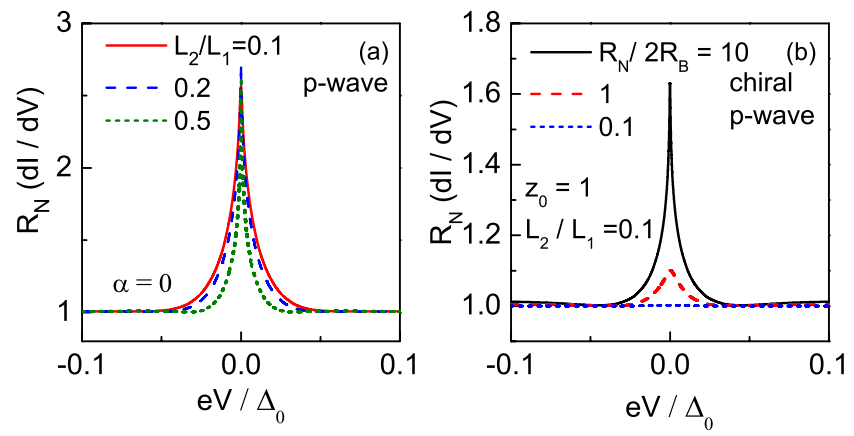

FIG. 2 (color online). Differential conductance in $p$-wave symmetry in (a) and chiral $p$-wave symmetry in (b). 
dent of $L_{2}$ as shown in Fig. 2(a) and follows from the analytic expression of the zero-bias conductance,

$$
\left.R_{N} \frac{d I}{d V}\right|_{e V=0}=\frac{\frac{R_{N} \cos \alpha}{2 R_{B} T_{B}}}{\tanh \left(\frac{R_{N} \cos \alpha}{2 R_{B} T_{B}}\right)} .
$$

The amplitude of the ZBCP decreases with the increase of the orientation angle $\alpha$ and vanishes at $\alpha=\pi / 2$. This is because the proximity effect is absent in a normal metal at $\alpha=\pi / 2[3,6]$. In the $p$-wave symmetry case, the ZBCP can be observed at temperatures below $E_{\text {th }}$ for almost all orientation angles. In Fig. 2(b), we discuss the conductance in the chiral $p$-wave symmetry to test realistic junctions involving $\mathrm{Sr}_{2} \mathrm{RuO}_{4}$ [1], where the form factor is given by $\Psi(\gamma)=\cos \gamma+i \sin \gamma$. The boundary condition in Eq. (8) can be used with $g_{s}=\left\{2 g+i\left(f_{1+} f_{2-}-f_{2+} f_{1-}\right)\right\}$, $f_{s}=-\left\{i g\left(f_{1+}-f_{1-}\right)+f_{2+}+f_{2-}\right\}, \quad \xi=1+$ $g^{2}+f_{1+} f_{1-}+f_{2+} f_{2-}, g=\epsilon / \sqrt{\epsilon^{2}-\Delta_{0}^{2}}$, and $f_{1(2), \pm}=$ $\operatorname{Re}(\operatorname{Im}) \Delta_{ \pm} / \sqrt{\Delta_{0}^{2}-\epsilon^{2}}[11]$. In the chiral $p$-wave junctions, the peak width is characterized also by $E_{\text {th }}$ but the zero-bias conductance is approximately given by Eq. (10) with $\cos \alpha / T_{B} \rightarrow 1$. In the limit of weak proximity effect such as $R_{N} / 2 R_{B}=0.1$, the $\mathrm{ZBCP}$ becomes small. In other cases, the proximity effect leads to the clear ZBCP as in Fig. 2(a). The conductance spectra in Figs. 2(a) and 2(b) show qualitatively similar behavior. In spin-triplet junctions, the boundary condition in Eq. (8) and the Usadel equation in Eq. (6) at $\epsilon=0$ yield the pure imaginary value of $\theta$ everywhere in a normal metal. Then the zero-bias anomaly in the conductance follows mathematically from this fact and Eq. (9) and therefore is the robust feature of the spin-triplet superconductors.

Next, let us summarize the differential conductance of the spin-singlet superconducting junctions in Fig. 3. In Fig. 3(a), the results for $s$-wave junctions are plotted for several choices of $R_{N} / 2 R_{B}$ at $z_{0}=0$. In contrast to the spin-triplet cases in Figs. 2, the conductance has the dip structure. In the spin-singlet junctions, the proximity effect has two contributions which influence the conductance in an opposite way. The induced superconductivity in a normal metal tends to assist electron transport. On the other
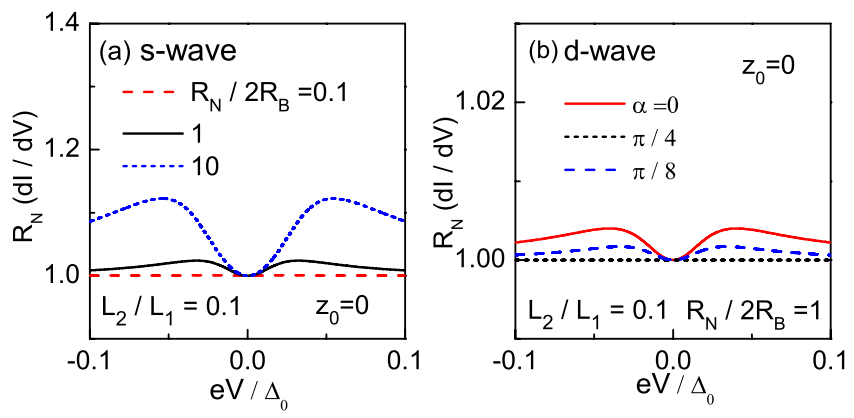

FIG. 3 (color online). Differential conductance in spin-singlet superconductor junctions for $s$-wave symmetry in (a) and $d$-wave symmetry in (b). hand, the existence of Cooper pairs decreases the DOS in a normal metal (the so-called minigap is formed), and this leads to the suppression of conductance. These two effects exactly cancel each other at $e V=0$ [13]. The positive contribution to conductance due the proximity effect decays in power law of $e V$, whereas the negative contribution decays exponentially [13]. Thus, the proximity effect slightly enhances the conductance around $e V \sim E_{\text {th }}$ and the conductance spectra show the dip structure as shown in Fig. $3[12,13]$. The degree of the enhancement depends on the strength of the proximity effect $\left(R_{N} / 2 R_{B}\right)$ and is typically of the order of percent as shown in (a). The characteristic behavior of the conductance is insensitive to $z_{0}$. In Fig. 3(b), we show the conductance in the $d$-wave symmetry for several choices of the orientation angle $\alpha$, where $z_{0}=0$ and $R_{N} / 2 R_{B}=1$. At $\alpha=0$, the conductance shows the dip structure near the zero bias as well as those in the $s$-wave junctions. The dip structure gradually disappears with the increase of $\alpha$. The conductance spectra become completely flat at $\alpha=\pi / 4$ because the proximity effect is absent in a normal metal [3,4]. In contrast to spintriplet junctions, Eqs. (6) and (7) at $\epsilon=0$ always yield a real value of $\theta$ for all spin-singlet junctions. This fact mathematically explains the cancellation of the two contributions of the proximity effect at $e V=0$ because Eq. (9) results in $d I / d V=R_{N}^{-1}$ for real $\theta$. Thus the dip structure around the zero bias in conductance spectra is the robust feature of spin-singlet superconductor junctions. This conclusion is valid only for the $T$-shaped junction in which a superconductor is away from the current path. In usual quasi-one-dimensional NS junctions, the proximity effect causes the ZBCP in the $s$-wave symmetry [19].

Here we explain the reasons for the zero-bias anomaly in spin-triplet junctions. Since electrons obey Fermi statistics, the pairing function of a Cooper pair satisfies the relation

$$
f_{\sigma, \sigma^{\prime}}(\boldsymbol{k}, \boldsymbol{\epsilon})=-f_{\sigma^{\prime}, \sigma}(-\boldsymbol{k},-\boldsymbol{\epsilon}),
$$

where $\sigma$ and $\sigma^{\prime}$ are the spin of the two electrons, $\boldsymbol{k}$ dependence of pairing function characterizes the symmetry of the orbital part. According to the relation, the Cooper pair in a superconductor is classified into spin-singlet evenparity and spin-triplet odd-parity symmetry classes. The interchange of spin (i.e., $\sigma \leftrightarrow \sigma^{\prime}$ ) and $\boldsymbol{k} \rightarrow-\boldsymbol{k}$ gives rise to the negative sign on the right-hand side of Eq. (11) in the former and in the latter, respectively. In a normal metal, only $s$-wave pairs are allowed irrespective of an original pairing symmetry in a superconductor because of the diffusive impurity scattering. In $p$-wave junctions, spin-triplet $s$-wave Cooper pairs penetrate into a normal metal. To satisfy Eq. (11), such Cooper pairs acquire the oddfrequency symmetry property [i.e., $f_{\sigma, \sigma^{\prime}}(\boldsymbol{k}, \boldsymbol{\epsilon})=$ $\left.-f_{\sigma, \sigma^{\prime}}(\boldsymbol{k},-\boldsymbol{\epsilon})\right]$. The most important feature of oddfrequency pairs is the enhancement of the quasiparticle DOS at $\epsilon=0[10,11]$. This feature is in contrast to the usual proximity effect in the spin-singlet junctions. Thus the proximity effect always increases the conductance in 
spin-triplet junctions. In addition to this, the DOS at $\epsilon=0$ becomes large because of the midgap Andreev resonant state (MARS) [20,21]. The large DOS at the Fermi energy is interpreted as the penetration of the MARS from a superconductor into a normal metal $[5,6]$. Thus the ZBCP in Fig. 2 reflects the peak structure of the DOS in a normal metal. The effects of MARS in the chiral $p$-wave symmetry are weaker than those in the $p$-wave symmetry because only quasiparticles with $\gamma=0$ contribute to the MARS. Thus the zero-bias conductance in the chiral $p$-wave junction is smaller than that in the $p$ wave as shown in Fig. 2. Odd-frequency symmetry compensates the symmetry change of the orbital part from odd-parity symmetry in a superconductor to the $s$-wave symmetry in a normal metal. Therefore we conclude that the ZBCP is expected in the $T$-shaped junctions of all spin-triplet superconductors.

Finally, we propose a new experiment to discriminate the symmetry of a superconductor. The proximity effect on remote current causes the clear-cut difference between the conductance spectra of the spin-triplet junctions and those in the spin-singlet ones, as shown in Figs. 2 and 3. Therefore the $T$-shaped junction can serve as a superconducting symmetry detector. In addition, the present proposal has several advantages compared to previous proposals $[5,6]$ with respect to resolving low energy transport. First, to observe the characteristic conductance spectra at $|e V| \lesssim E_{\text {th }}$, it is necessary to suppress the influence of undesired scattering due to defects and/or localized states at the NS interface because the tunneling conductance is extremely sensitive to interface quality. The tunneling current through such states easily washes out the expected conductance signals. In fact, the bad interface quality damages the subgap tunneling spectra of $\mathrm{Sr}_{2} \mathrm{RuO}_{4}$. The conductance of the $T$-shaped geometry, however, is rather insensitive to interface quality because current does not flow through the NS interface. Second, within the present technologies it is difficult to realize small and highly transparent NS junctions for observing the Josephson current. This is because the unconventional superconductors are usually synthesized as bulk materials and are not suitable for microfabrication. The $T$-shaped junctions, however, require microfabrication only on the normal metal (not on a superconductor). Thus the $T$-shaped junctions are accessible within the present technique. Finally the proposed experiment can test ferromagnetic superconductors because the measurement of conductance spectra does not require an external magnetic field. For these reasons, we conclude that the $T$-shaped junctions would be a powerful tool to test the symmetry of superconductors.

In conclusion, we have studied the conductance spectra of $T$-shaped superconductor junctions. The proximity effect on the remote current modifies low energy transport depending remarkably on the symmetry of superconductors. In the case of spin-triplet superconductors, the conductance shows the zero-bias anomaly. The odd-frequency
Cooper pairs in a normal metal cause the anomaly and the midgap Andreev resonant states support the robustness of this drastic effect. In contrast to the spin-triplet case, conductance spectra in spin-singlet junctions always show the dip structure around the zero bias. On the basis of calculation results, we have proposed a new experimental method to detect spin-triplet superconductivity and have discussed the advantages of the method.

This work was partially supported by the Dutch FOM, the NanoNed program under Grant No. TCS7029, and a Grant-in-Aid for Scientific Research from The Ministry of Education, Culture, Sports, Science and Technology of Japan (Grants No. 18043001, No. 17071007, No. 19540352, and No. 17340106).

[1] A. P. Mackenzie and Y. Maeno, Rev. Mod. Phys. 75, 657 (2003).

[2] S.S. Saxena et al., Nature (London) 406, 587 (2000); D. Aoki et al., Nature (London) 413, 613 (2001).

[3] Y. Asano, Phys. Rev. B 64, 014511 (2001); J. Phys. Soc. Jpn. 71, 905 (2002).

[4] Y. Tanaka, Yu. V. Nazarov, and S. Kashiwaya, Phys. Rev. Lett. 90, 167003 (2003).

[5] Y. Asano, Y. Tanaka, and S. Kashiwaya, Phys. Rev. Lett. 96, 097007 (2006).

[6] Y. Tanaka and S. Kashiwaya, Phys. Rev. B 70, 012507 (2004); Y. Tanaka, T. Yokoyama, and S. Kashiwaya, Phys. Rev. B 71, 094513 (2005).

[7] Y. Tanaka and A. A. Golubov, Phys. Rev. Lett. 98, 037003 (2007).

[8] F. S. Bergeret, A. F. Volkov, and K. B. Efetov, Phys. Rev. Lett. 86, 4096 (2001); Rev. Mod. Phys. 77, 1321 (2005); Physica (Amsterdam) 367C, 107 (2002).

[9] R.S. Keizer, S. T.B. Goennenwein, T. M. Klapwijk, G. Miao, G. Xiao, and A. Gupta, Nature (London) 439, 825 (2006).

[10] Y. Asano, Y. Tanaka, and A. A. Golubov, Phys. Rev. Lett. 98, 107002 (2007).

[11] Y. Tanaka, Y. Asano, A. A. Golubov, and S. Kashiwaya, Phys. Rev. B 72, R140503 (2005).

[12] Yu. V. Nazarov and T. H. Stoof, Phys. Rev. Lett. 76, 823 (1996).

[13] A. F. Volkov and H. Takayanagi, Phys. Rev. Lett. 76, 4026 (1996); Phys. Rev. B 56, 11184 (1997).

[14] K. Usadel, Phys. Rev. Lett. 25, 507 (1970).

[15] W. Belzig, F. K. Wilhelm, C. Bruder, and G. Schön, Superlattices Microstruct. 25, 1251 (1999).

[16] A. F. Volkov, A. V. Zaitsev, and T. M. Klapwijk, Physica (Amsterdam) 210C, 21 (1993).

[17] A. V. Zaitsev, Phys. Lett. A 194, 315 (1994).

[18] Yu. V. Nazarov, Phys. Rev. Lett. 73, 1420 (1994); Superlattices Microstruct. 25, 1221 (1999).

[19] A. Kastalsky, A. W. Kleinsasser, L. H. Greene, R. Bhat, F. P. Milliken, and J. P. Harbison, Phys. Rev. Lett. 67, 3026 (1991).

[20] C. R. Hu, Phys. Rev. Lett. 72, 1526 (1994).

[21] Y. Tanaka and S. Kashiwaya, Phys. Rev. Lett. 74, 3451 (1995). 